\title{
Avaliação da Estabilidade Fenotípica para Produção de Leite em Animais da Raça Holandesa
}

\author{
Douglas Messias Lamounier Camargos Rezende1, Daniel Furtado Ferreira², Tarcisio de \\ Moraes Gonçalves ${ }^{3}$
}

RESUMO - O objetivo deste trabalho foi estimar os parâmetros de estabilidade fenotípica da produção de leite, a partir de dados cedidos pela Associação de Criadores de Gado Holandês de Minas Gerais (ACGHMG), envolvendo animais de diferentes grupamentos genéticos da raça Holandesa (31/32, GC1, GC2, GC3, \GC4 e PO), diferentes núcleos (15, 25, 35, 45, 55, 65 e 75) e dois níveis de manejo. O banco de dados ficou limitado a 22.560 lactações até 305 dias, ocorridas no período de 1989 a 1996. Animais cuja duração de lactação foi menor que 150 dias e/ou produção até 305 dias foi menor que $750 \mathrm{~kg}$ de leite e/ou tiveram causa de secagem anormal foram eliminados. Para se estudar a interação genótipo x ambiente, foi aplicada a metodologia proposta por TOLER (1990). Os grupamentos genéticos 31/ 32 e GC1 ( $1^{\text {a }}$ geração controlada) apresentaram padrão de resposta linear frente à variação do ambiente. Ambos foram separados como genótipos de baixo nível de produção e classificados segundo a metodologia de TOLER (1990) nos grupos D e C, respectivamente. Os genótipos GC2 ( $2^{\mathrm{a}}$ geração controlada), GC3 ( $3^{\mathrm{a}}$ geração controlada), $\geq \mathrm{GC} 4$ ( da $4^{\mathrm{a}}$ à $11^{\mathrm{a}}$ geração controlada) e PO (puro de origem) apresentaram modelos bisegmentados. Estes grupamentos genéticos foram separados como de alto nível de produção e classificados, respectivamente, como pertencentes aos grupos A, F, E e G (Toler, 1990). O genótipo GC2 (2ª geração controlada) obteve o melhor desempenho, quanto ao nível de produção, e foi classificado no grupo A, podendo ser recomendado para todos os tipos de ambiente.

Palavras-chave: estabilidade fenotípica, raça holandesa, produção de leite

\section{Evaluation of the Phenotypic Stability for Milk Production in Dairy Cattle of Holstein Breed}

\begin{abstract}
This work was carried out to estimate stability parameters of milk production using data from "Associação de Criadores de Gado Holandês de Minas Gerais (ACGHMG)". Animals from different genetic groups of the Holstein breed (31/32, GC1, GC2 GC3, $\geq \mathrm{GC} 4$ and PO), with different regions $(15,25,35,45,55,65$ and 75$)$ and with two management systems. The database was defined by 22560 animals milk production up to 305 days, from 1989 to 1996. The following animals criteria was used to discord animals for the analyses: suckling smaller than 150 days or production up to 305 days, smaller than $750 \mathrm{~kg}$ of milk, or animals with abnormal drying were eliminated. The TOLER (1990) methodology was applied to study the genotype $x$ environment interaction. The genotype $31 / 32$ and GC1 ( $1^{\text {st }}$ controlled generation) presented linear patterns of response across environments. Both were separated as genotypes of low milk production level, and they were classified according to TOLER (1990) in the groups D and C, respectively. The genotypes GC2 (2nd controlled generation), GC3 ( $3^{\text {rd }}$ controlled generation), $\geq \mathrm{GC} 4$ (of the $4^{\text {th }}$ to $11^{\text {th }}$ controlled generations) and PO (pure of origin) presented bi-segmented models with different inclinations. All of theses genotypes were separate as of high production level. According to TOLER (1990), they were classified as belonging to the groups A, F, E and G, respectively. The results allowed that genotype GC2 ( $2^{\text {nd }}$ controlled generation) was included in the group A, with high milk production, and it could be recommended for favorable or unfavorable environments.
\end{abstract}

Key Words: phenotypic stability, Holstein breed animals, milk production

\section{Introdução}

Antes que uma raça ou animais de diferentes grupamentos genéticos sejam recomendados, o melhorista necessita fazer avaliações em vários locais, durante vários anos e condições de manejo. Esta fase, além de ser a mais onerosa, requer muita precisão dos experimentos. As avaliações em vários ambientes, embora permitam que os grupamentos mais produtivos sejam identificados, necessitam de análise estatística mais criteriosa que a análise de variância conjunta para que os com menor oscilação de produção, ou de maior estabilidade fenotípica, possam ser detectados. Deseja-se que os grupamentos genéticos potencialmente superiores sejam uniformemente superiores em todos os ambientes (HAMILTON et al., 1996). Falha na classificação consistente do desempenho genotípico de um ambiente para

\footnotetext{
${ }^{1}$ Acadêmico do décimo módulo de Zootecnia da Universidade Federal de Lavras, Bolsista do CNPq em IC.

2 Professor Dr. Departamento de Ciências Exatas da UFLA, e-mail: danielff(@ufla.br, Pesquisador Bolsista do CNPq.

${ }^{3}$ Professor do Departamento de Zootecnia da UFLA.
} 
outro é conhecida como interação genótipo x ambiente (WESTCOTT, 1986).

O aproveitamento da interação é uma estratégia utilizada pelos melhoristas. Quando ocorrer a interação, deverão ser selecionados grupamentos genéticos específicos para uso em ambientes específicos. Esta estratégia é limitada pelo amplo espectro de ambientes encontrados nos países de clima tropical.

Uma segunda estratégia baseia-se na seleção de reduzido número de genótipos para uso em todos os ambientes de interesse, com padrão de performance aceitável para ambientes desfavoráveis, mas que sejam responsivos à melhoria das condições de manejo. Muitas metodologias que foram desenvolvidas e discutidas na literatura, com a finalidade de avaliar o padrão de performance genotípica nestes ambientes, vem sendo largamente aplicadas ao melhoramento de plantas (LIN et al., 1986; WESTCOTT, 1986; BECKER e LEON, 1988; e CROSSA, 1990). No melhoramento animal não se encontram relatos de sua utilização no país, embora existam estudos relatando o efeito da interação genótipo $\mathrm{x}$ ambiente sobre a produção de leite da raça holandesa (RORATO et al., 1994; HOHENBOKEN, 1996).

Os experimentos para avaliar a interação genótipo $\mathrm{x}$ ambiente, apesar de grande importância, não fornecem informações pormenorizadas sobre o comportamento de cada genótipo frente às variações ambientais. As estratégias que permitem fazer previsão do desempenho dos genótipos de comportamento estável e capazes de serem responsivos às variações ambientais são denominadas de análises de adaptabilidade e estabilidade (PLAISTED e PATERSON, 1959; EBERHART e RUSSELL, 1966; VERMA, CHAHAL e MURTY, 1978; LIN e BINNS, 1988; e CRUZ et al., 1989).

Entre as várias críticas relacionadas às várias metodologias de análise de estabilidade, a principal refere-se ao fato de que os índices ambientais utilizados não são independentes nas metodologias que envolvem regressão linear, que representam a maioria dos casos. Alternativas para resolver este problema foram apresentadas por FERREIRA et al. (1992), os quais utilizaram a performance média de genótipos testemunhas como índice ambiental independente. Esta metodologia tem limitações com relação à escolha das testemunhas. Mais recentemente, os melhoristas têm dado atenção a métodos multivariados, como a técnica AMMI (Additive Main effects and Multiplicative Interaction effects), envolvendo componentes principais e considerando a interação como efeito multiplicativo (GAUCH JR. e ZOBEL, 1988;
CROSSA, 1990). Esta metodologia, embora elogiada por alguns, tem mostrado resultados insatisfatórios, quando o componente principal não explica um percentual superior a $70 \%$ da variação, devido à interação (ARIAS, 1996).

Uma metodologia que avalia o padrão de comportamento genotípico frente ao ambiente, por meio de um modelo e testes que preservam as exigências estatísticas, independência do índice ambiental, exigida nas aproximações por regressão, foi apresentada por TOLER (1990) e TOLER e BURROWS (1998). Trata-se de um modelo não-linear nos parâmetros, apresentado nas versões linear simples, análogo ao modelo de EBERHART e RUSSELL (1966), e linear bissegmentado, análogo ao modelo de CRUZ et al. (1989). Os resultados fornecidos por estes modelos são utilizados juntamente com a performance média de cada grupamento, com a finalidade de agrupá-los de acordo com o seu padrão de resposta ao ambiente. Esta metodologia fundamenta-se basicamente no fato de que o índice ambiental é um parâmetro a ser estimado e que os modelos são não lineares nos parâmetros, exigindo métodos mais refinados de estimação em relação aos quadrados mínimos ordinários, como por exemplo, o método de Gauss Newton modificado (GALLANT, 1987).

O modelo 1 de Toler, que prevê comportamento linear para os genótipos frente à variação ambiental, é dado por:

$$
Y_{i j}=\alpha_{i}+\beta_{i} \mu_{j}+\delta_{i j}+\varepsilon_{i j}
$$

em que $Y_{i j}$ é a média de produção do i-ésimo genótipo noj-ésimo ambiente; $\alpha_{i}$, a média de produção do i-ésimo genótipo; $\beta_{i}$, o coeficiente de sensibilidade de resposta ao ambiente do i-ésimo genótipo; e $\mu_{j}$, o índice ambiental; $\delta_{i j}$, o desvio de regressão do i-ésimo genótipo no j-ésimo ambiente; e $\varepsilon_{i j}$, o efeito do erro médio associado à observação $Y_{i j} ; \mathrm{i}=1,2, \ldots, \mathrm{p}$ e j=1, $2, \ldots$, q. A única diferença deste modelo em relação ao de EBERHART e RUSSELL (1966) é que o índice ambiental deve ser estimado, sendo, portanto, um parâmetro do modelo, juntamente com os $\alpha_{i}$ 's e $\beta_{i}$ 's, sujeitos às restrições $\sum_{i=1}^{p} \beta_{i}=p$ e $\sum_{j=1}^{q} \mu_{j}=0$.

O modelo 2 de Toler (1990), similar ao de CRUZ et al. (1989), é apresentado a seguir:

$$
Y_{i j}=\alpha_{i}+\left[\beta_{1 i} Z_{j}+\beta_{2 i}\left(1-Z_{j}\right)\right]+\delta_{i j}+\varepsilon_{i j}
$$

em que $Y_{i j}$ é a média de produção do i-ésimo genótipo no j-ésimo ambiente; $\alpha_{i}$ representa o inter- 
cepto de resposta do i-ésimo genótipo; $\beta_{1 i}$ e $\beta_{2 i}$ representam os coeficientes de sensibilidade de resposta do i-ésimo genótipo em ambientes desfavorável e favorável, respectivamente; $\mu_{j}$ representa o efeito ambiental; $\delta_{i j}$ é o desvio de regressão do i-ésimo genótipo no j-ésimo ambiente; $\mathcal{E}_{i j}$ é o efeito do erro médio associado à observação $Y_{i j} ; \mathrm{i}=1,2, \ldots, \mathrm{p}$ e j= $1,2, \ldots, \mathrm{q} ; \mathrm{Zj}=1$ se $\mu_{j} \leq 0$, e $Z \mathrm{j}=0$ se $\mu_{j}>0$. As restrições impostas a este modelo são:

$$
\sum_{i=1}^{p} \beta_{1 i}=\sum_{i=1}^{p} \beta_{2 i}=p_{\text {e }} \sum_{j=1}^{q} \mu_{j}=0
$$

Os critérios de classificação dos genótipos, de acordo com o padrão de resposta aos ambiente, foi proposto por Toler (1990) e está apresentado na Tabela 1. O significado prático da classificação está apresentado na Tabela 2 .

Esta classificação (Tabelas 1 e 2) refere-se somente ao padrão de comportamento dos genótipos com a variação ambiental, sendo insuficiente pelo fato de não se considerar a performance genotípica média. Critérios de seleção entre os genótipos devem combinar a classificação quanto ao padrão de respos- ta frente à variação ambiental com a produtividade média dos grupamentos genéticos.

O objetivo deste trabalho foi estimar os parâmetros de estabilidade da produção de leite, a partir de dados cedidos pelo serviço de controle leiteiro da Associação de Criadores de Gado Holandês de Minas Gerais (ACGHMG), envolvendo animais de diferentes grupamentos genéticos, utilizando-se os modelos de Toler (1990).

\section{Material e Métodos}

O banco de dados utilizado para os testes requeridos pelo experimento, ficou limitado a 22.560 lactações com duração até 305 dias, ocorridas entre os anos de 1989 a 1996. Foram utilizados animais de seis grupamentos genéticos: $31 / 32, \mathrm{GC} 1\left(1^{\mathrm{a}}\right.$ geração controlada), GC2 (2a geração controlada), GC3 (3 ${ }^{\mathrm{a}}$ geração controlada), $\geq \mathrm{GC} 4$ definido como agrupamento das gerações controladas restantes (GC4 a GC11) e PO. Consideraram-se dois níveis de manejo: nível de manejo alto e baixo e sete núcleos (regiões), que foram representados pelos seus números: 15, 25,

Tabela 1 - Critérios de classificação genotípica, de acordo com o padrão de resposta aos ambientes Table 1 - Environment patterns to genotype classification criterion

\begin{tabular}{|c|c|}
\hline Grupo & Critério de grupamento \\
\hline Group & Group criterion \\
\hline A & Rejeitar a hipótese (reject the hypothesis) $\beta_{1}=\beta_{2}$ e $\beta_{1}<1<\beta_{2}$ \\
\hline B & $\begin{array}{l}\text { Aceitar a hipótese (accept the hypothesis) } \beta_{1}=\beta_{2} \text {, rejeitar (reject) } \beta=1 \\
\text { e } \beta>1 \text { (comum - common) }\end{array}$ \\
\hline $\mathrm{C}$ & Aceitar a hipótese (accept the hypothesis) $\beta_{1}=\beta_{2}$, aceitar (accept) $\beta=1$ \\
\hline $\mathrm{D}$ & $\begin{array}{l}\text { Aceitar a hipótese (accept the hypothesis) } \beta_{1}=\beta_{2} \text {, rejeitar (reject) } \beta=1 \\
\text { e } \beta>1 \text { (comum - common) }\end{array}$ \\
\hline $\mathrm{E}$ & Rejeitar a hipótese (reject the hypothesis) e $\beta_{1}=\beta_{2}$ e $\beta_{1}>1>\beta_{2}$ \\
\hline
\end{tabular}

Tabela 2 - Significado prático da classificação genotípica, de acordo com o padrão de resposta aos ambientes Table 2 - Practical meaning of the environment patterns to genotype classification criterion

\begin{tabular}{|c|c|}
\hline Grupo & Significado prático da resposta aos ambientes \\
\hline Group & Means of response patterns to environments \\
\hline$\overline{\mathrm{A}}$ & Resposta convexa e duplamente desejável \\
\hline & Convex response patterns and double desirable \\
\hline B & $\begin{array}{l}\text { Resposta linear simples e desejável só em ambientes de alta qualidade } \\
\text { Linear response patterns and desirable to high quality environments }\end{array}$ \\
\hline $\mathrm{C}$ & $\begin{array}{l}\text { Resposta linear simples, não desviando da resposta média } \\
\text { Linear response patterns and average response }\end{array}$ \\
\hline $\mathrm{D}$ & $\begin{array}{l}\text { Resposta linear simples e desejável só em ambientes de baixa qualidade } \\
\text { Linear response patterns and desirable to lower quality environments }\end{array}$ \\
\hline $\mathrm{E}$ & $\begin{array}{l}\text { Resposta côncava e duplamente indesejável } \\
\text { Concave response patterns and double undesirable }\end{array}$ \\
\hline
\end{tabular}


$35,45,55,65$ e 75 . Considerou-se para a execução dos testes catorze ambientes (combinações entre os sete núcleos e dois níveis de manejo).

A média de produção de leite até 305 dias, para as vacas que pariram com até 42 meses de idade, foi o critério utilizado para classificar os grupamentos genéticos em dois grupos quanto a média de produção (baixa $=$ rebanhos com produção de leite menor que $5.002,56 \mathrm{~kg}$; e alta $=$ rebanhos com produção de leite maior ou igual a $5.002,56 \mathrm{~kg}$ de leite).

Animais com duração de lactação inferior a 150 dias e produção menor que $750 \mathrm{~kg}$ foram eliminados das análises. Quando a lactação se estendeu por mais de 305 dias, considerou-se a produção obtida até 305 dias. Foram utilizados somente animais cujas produções foram obtidas em duas ordenhas. Animais que apresentaram causas de secagem anormais foram eliminados.

Foram consideradas duas estações de parição distintas: a estação seca e a das águas. A estação da seca foi definida no período de abril a setembro e a das águas, no período de outubro a março. Procurouse neste trabalho estudar somente os animais da raça holandesa presentes nos registros da ACGHMG.

Inicialmente foi realizada a análise de variância dos dados da produção leiteira, para avaliar o efeito da interação genótipo $\mathrm{x}$ ambientes. O modelo estatístico adotado contemplou os efeitos de grupamento genético, núcleo, nível de manejo, estação e ano de parição, bem como os efeitos de interação entre os efeitos principais. Utilizou-se ainda a covariável idade do primeiro parto considerando o efeito linear e quadrático. As análises de variância foram realizadas no sistema $\mathrm{SAS}^{\circledR}$ (Statistical analysis system), proc GLM (General Linear Models), SAS Institute (1995), devido ao grande desbalanceamento. As médias ajustadas (LSMEANS - Least Squares Means) dos grupamentos genéticos nos ambientes (nível de manejo e núcleos) foram solicitadas para a análise de estabilidade fenotípica.

A partir das médias ajustadas, foram realizadas as análises requeridas pelo método de Toler (1990), utilizando o software Toler (FERREIRA e ZAMBALDE, 1997). Este programa realizou a estimação dos parâmetros (coeficientes do modelo de regressão e índice ambiental) de estabilidade fenotípica pelo método de quadrados mínimos para modelos não-lineares (GALLANT, 1987). Para isso foi utilizado o processo de Gauss Newton modificado.

\section{Resultados e Discussão}

O resumo da análise de variância para avaliação do efeito da interação genótipo x ambiente está apresentado na Tabela 3. Verifica-se que houve efeito significativo $(\mathrm{P}<0,01)$ de grupamento genético e ambiente (núcleo e nível de manejo). As interações de grupamento genético com núcleo e com nível de manejo, bem como a interação tripla, foram significativas $(\mathrm{P}<0,01)$. Apesar de a precisão experimental ter sido considerada baixa, refletida pelo coeficiente de variação de $27,4 \%$, foi detectado efeito significativo das interações entre grupamento genético e os efeitos ambientais (núcleo e nível).

Tabela 3 - Análise de variância ambiental conjunta dos grupamentos genéticos para produção de leite em até 305 dias

Table 3 - Group analysis of variance to the genotype group over environments for milk production days up to 305

\begin{tabular}{|c|c|c|}
\hline Fator de variação & $\overline{\mathrm{gl}}$ & Quadrado médio $\times 10^{6}$ \\
\hline Source of variation & $d f$ & Mean square $\times 10^{6}$ \\
\hline Núcleo (Region) & 6 & $131^{* *}$ \\
\hline Nível (Level) & 1 & $1007 * *$ \\
\hline Grupo genético (Genetic group) & 5 & $77,31 * *$ \\
\hline $\begin{array}{l}\text { Nível x grupo genético } \\
\text { Level x genetic group }\end{array}$ & 5 & $36,88^{* *}$ \\
\hline $\begin{array}{l}\text { Núcleo } \mathrm{x} \text { grupo genético } \\
\text { Region } x \text { genetic group }\end{array}$ & 30 & $13,07^{* *}$ \\
\hline $\begin{array}{l}\text { Núcleo x Nível x Grupo genético } \\
\text { Region } x \text { Level x Genetic group }\end{array}$ & 36 & $19,05^{* *}$ \\
\hline $\begin{array}{l}\text { Erro } \\
\text { Error }\end{array}$ & 22461 & 2,055 \\
\hline $\begin{array}{l}\text { CV } \\
\text { Média (x) em kg até } 305 \text { dias } \\
\text { Mean in kg until } 305 \text { days }\end{array}$ & & $\begin{array}{c}27,4 \% \\
5002,56\end{array}$ \\
\hline
\end{tabular}


Por meio do modelo 2 de Toler, modelo bissegmentado, foi realizado o teste da hipótese da igualdade dos coeficientes de sensibilidade de respostas dos grupamentos aos ambientes favoráveis e desfavoráveis (Tabela 4). Verificou-se que os grupamentos genéticos 31/32 e GC1 apresentaram comportamento linear. Os grupamentos genéticos $\mathrm{GC} 2, \mathrm{GC} 3$ e $\geq \mathrm{GC} 4$ apresentaram padrão de resposta não-linear $(\mathrm{P}<0,01)$, o que ocorreu também para o PO $(\mathrm{P}<0,05)$.

As estimativas de $\beta_{1}$ e $\beta_{2}$, coeficientes de sensibilidade de respostas para os ambientes desfavoráveis e favoráveis, respectivamente, estão apresentados na Tabela 5, para os genótipos GC2, GC3, $\geq \mathrm{GC} 4$ e PO. Para os ambientes desfavoráveis, verifica-se que os genótipos GC2 e GC3 apresentaram estimativas significativamente menores que $1(\mathrm{P}<0,01)$, e os genótipos $\geq \mathrm{GC} 4$ e $\mathrm{PO}$, estimativas de coeficiente angular significativamente maiores que 1 .

As hipóteses de que os coeficientes de sensibilidade de respostas dos ambientes favoráveis é igual a 1 foi rejeitada para o GC2 e o $\geq \mathrm{GC} 4$. O grupamento genético GC2 apresentou $\beta_{2}>1$ e o $\geq$ GC4, $\beta_{2}<1$.
Os genótipos GC3 e PO apresentaram resposta média aos ambientes favoráveis $\left(\beta_{2}=1\right)$. $\mathrm{O}$ genótipo $\geq \mathrm{GC} 4$ não deve ser recomendado a ambientes favoráveis, por não ser responsivo $\left(\beta_{2}<1\right)$, já o GC2 foi altamente responsivo à melhoria das condições ambientais $\left(\beta_{2}>1\right)$.

$\mathrm{Na}$ Tabela 6 são apresentados as estimativas do coeficiente angular comum, bem como o seu erro padrão e teste para a hipótese $\mathrm{H}_{\mathbf{0}}: \beta=\mathbf{1}$, somente para os grupamentos genéticos com comportamento linear, que foram 31/32 e GC1. Verificou-se que apenas o grupamento genético $31 / 32$ apresentou significativamente inferior a 1. Concluiu-se que este material só pode ser utilizado em ambientes desfavoráveis ou de baixa qualidade. É oportuno salientar que os ambientes considerados desfavoráveis são todos aqueles de qualidade inferior aos normalmente utilizados para os rebanhos de elite, por se referirem ao efeito conjunto de clima e manejo. O genótipo GC1 teve resposta linear simples, não desviando da resposta média $(\beta=1)$.

Na Tabela 7 estão apresentados os valores de $\alpha$, de acordo com os modelos linear simples e bissegmentado para cada grupamento genético. Ve-

Tabela 4 -Estimativas de $\beta_{2}$ e $\beta_{1}$ e erro-padrão para os grupamentos genéticos dos animais da raça holandesa

Table 4 - Estimates of $\beta_{2}$ and $\beta_{1}$ and standard errors for genetic groups of Holstein breed animals

\begin{tabular}{ccc}
\hline $\begin{array}{l}\text { Grupamento genético } \\
\text { Genetic group }\end{array}$ & $\begin{array}{c}\text { Estimativa } \beta_{2}=\beta_{1} \\
\text { Estimate }\end{array}$ & $\begin{array}{c}\text { Erro-padrão } \\
\text { Standard error }\end{array}$ \\
\hline $31 / 32$ & $-0,3245$ & 0,4443 \\
GC1 & $-0,0993$ & 0,4426 \\
GC2 & $1,6537^{* *}$ & 0,4707 \\
GC3 & $1,4158^{* *}$ & 0,4695 \\
$\geq$ GC4 & $-1,5390^{* *}$ & 0,4696 \\
PO & $-1,1065^{*}$ & 0,4622 \\
\hline
\end{tabular}

* Significativo pelo teste t a $5 \%$ (Significant $(P<.05)$ by $t$ test).

** Significativo pelo teste $t$ a $1 \%$ (Significant $(P<.01)$ by $t$ test).

Tabela 5 - Estimativas de $\beta_{2}$ e $\beta_{1}$ para os grupamentos genéticos com modelo bissegmentado e o teste de igualdade destes parâmetros a 1

Table 5 - Estimates of $\beta_{2}$ and $\beta_{1}$ to genetic groups with bi segmented models and the hypothesis tests of equal parameter value to 1

\begin{tabular}{cccc}
\hline $\begin{array}{c}\text { Grupamento genético } \\
\text { Genetic group }\end{array}$ & $\begin{array}{c}\text { Parâmetro } \\
\text { Parameter }\end{array}$ & $\begin{array}{c}\text { Estimativa } \\
\text { Estimate }\end{array}$ & $\begin{array}{c}\text { Erro-padrão } \\
\text { Standard error }\end{array}$ \\
\hline GC2 & $\beta_{1}$ & $0,1651^{* *}$ & 0,2826 \\
& $\beta_{2}$ & $1,8188^{* *}$ & 0,2242 \\
$\mathrm{GC} 3$ & $\beta_{1}$ & $-0,0482^{* *}$ & 0,2849 \\
& $\beta_{2}$ & 1,3676 & 0,2210 \\
$\mathrm{GC} 4$ & $\beta_{1}$ & $1,9564^{* *}$ & 0,2836 \\
& $\beta_{2}$ & $0,4174^{* *}$ & 0,2220 \\
$\mathrm{PO}$ & $\beta_{1}$ & $1,9326^{* *}$ & 0,2807 \\
& $\beta_{2}$ & 0,8261 & 0,2185 \\
\hline
\end{tabular}

${ }^{* *}$ significativo pelo teste $t$ a $1 \%$ (Significant $(P<.01)$ by $t$ test). 
rificou-se que os grupamentos genéticos $31 / 32$ e GC1 foram os que apresentaram menores valores de $\alpha$, sendo classificado como de baixa média de produção. O 31/32 foi classificado anteriormente como não responsivo à melhoria do ambiente e, como está associado à baixa produção média, é um grupamento genético cuja utilização não deve ser recomendada. Com base nestes resultados, foram obtidos o padrão de classificação genotípica frente à variação ambiental proposta por TOLER (1990) e a classificação quanto aos níveis de produção dos grupamentos genéticos (Tabela 8). É conveniente salientar que para o grupamento genético $\mathrm{GC} 3$, de alta produção, por apresentar comportamento linear bissegmentado, com $\beta_{1}<1$ e $\beta_{2}=1$, não foi encontrado um padrão de classificação (Tabela 1) proposto por Toler (1990). Concluiu-se que seria necessário propor um novo grupo, denominado de "F", para os grupamentos genéticos desejáveis para ambientes desfavoráveis $\left(\beta_{1}<1\right)$ e com responsividade média à melhoria das condições ambientais $\left(\beta_{2}=1\right)$, em ambientes favoráveis.

Para o grupamento genético PO, de maior média de produção e com $\beta_{1}>1$ e $\beta_{2}=1$, também não existe um padrão de classificação para a proposta de Toler (1990). Dessa forma, foi necessário criar novo grupo "G", para os genótipos com $\beta_{1}>1$, não desejáveis para ambientes desfavoráveis, porém com resposta média $\left(\beta_{2}=1\right)$ aos ambientes favoráveis. Verificou-se

Tabela 6 - Estimativas de $\beta$ para os grupamentos genéticos com modelo linear e teste $\mathrm{H}_{0}: \beta=1$

Table 6 - Estimates of $\beta$ to the genetic groups with linear model and the hypotheses tests $\mathrm{H}_{0}: \beta=1$

\begin{tabular}{lcc}
\hline $\begin{array}{l}\text { Grupamento genético } \\
\text { Genetic group }\end{array}$ & $\begin{array}{c}\text { Estimativa de } \beta \\
\text { Estimate of } \beta\end{array}$ & $\begin{array}{c}\text { Erro-padrão } \\
\text { Standard error }\end{array}$ \\
\hline $31 / 32$ & $0,7940^{*}$ & 0,0921 \\
$\mathrm{GCl}$ & 0,9449 & 0,0918 \\
\hline
\end{tabular}

*significativo pelo teste $\mathrm{t}$ a $5 \%$ (Significant $(P<.05)$ by $t$ test).

Tabela 7 - Estimativas dos $\alpha$ para cada grupamento genético, para os modelos linear simples e bissegmentado

Table 7 - Estimates of $\alpha$ for each genetic groups with linear and bi segmented models

\begin{tabular}{|c|c|c|c|c|}
\hline \multirow[t]{2}{*}{$\begin{array}{l}\text { Grupamento genético } \\
\text { Genetic group }\end{array}$} & \multicolumn{2}{|c|}{$\begin{array}{l}\text { Modelo simples } \\
\text { Linear model }\end{array}$} & \multicolumn{2}{|c|}{$\begin{array}{c}\text { Modelo bissegmentado } \\
\text { Bi segmented model }\end{array}$} \\
\hline & $\begin{array}{l}\text { Estimativa } \\
\text { Estimate }\end{array}$ & $\begin{array}{l}\text { Erro padrão } \\
\text { Standard error }\end{array}$ & $\begin{array}{l}\text { Estimativa } \\
\text { Estimate }\end{array}$ & $\begin{array}{l}\text { Erro-padrão } \\
\text { Standarderror }\end{array}$ \\
\hline $31 / 32$ & 4713,9 & 70,7 & 4816,0 & 156,7 \\
\hline $\mathrm{GCl}$ & 4973,5 & 70,7 & 5004,8 & 156,2 \\
\hline $\mathrm{GC} 2$ & 5397,7 & 70,7 & 4877,3 & 165,7 \\
\hline GC3 & 5269,0 & 70,7 & 4823,5 & 164,9 \\
\hline$\geq \mathrm{GC} 4$ & 5325,3 & 70,7 & 5809,6 & 165,1 \\
\hline $\mathrm{PO}$ & 5509,4 & 70,7 & 5857,6 & 162,4 \\
\hline
\end{tabular}

Tabela 8 - Classificação dos grupamentos genéticos quanto a seu padrão de resposta ao ambiente e quanto à média de produção

Table 8 - Environments patterns and yield performance classifications for genotypes

\begin{tabular}{|c|c|c|c|c|c|c|c|}
\hline $\begin{array}{l}\text { Média de produção } \\
\text { Yield average }\end{array}$ & $\mathrm{A}$ & B & $\bar{C}$ & $\mathrm{D}$ & $\mathrm{E}$ & $\bar{F}$ & $\mathrm{G}$ \\
\hline Alta & $\mathrm{GC} 2$ & - & - & - & $\geq \mathrm{GC4}$ & GC3 & $\mathrm{PO}$ \\
\hline High & & & & & & & \\
\hline $\begin{array}{l}\text { Baixa } \\
\text { Low }\end{array}$ & - & - & $\mathrm{GCl}$ & $31 / 32$ & - & - & - \\
\hline
\end{tabular}


Rev. bras. zootec.

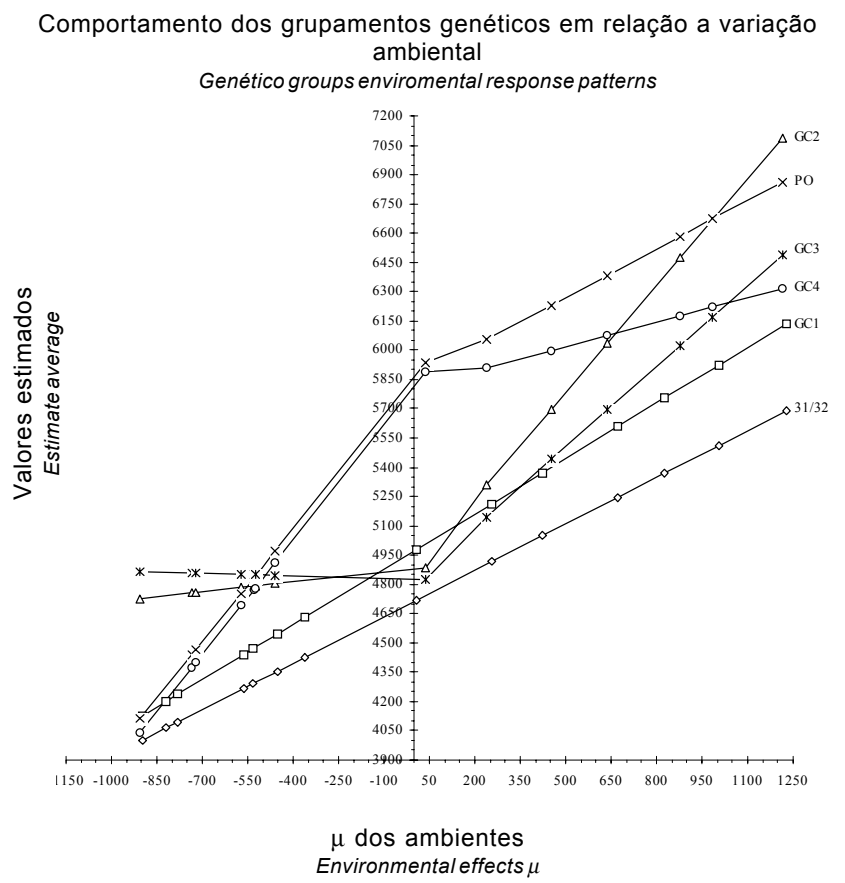

Figura 1 - Padrão de resposta ao ambiente quanto à produção em quilos de leite em até 305 dias para os seis genótipos nos 14 ambientes estudados.

Figure 1 - Environment response pattern to milk productions in up to 305 days for 6 genotypes across 14 environments. que o grupamento genético GC2 apresentou produção alta e foi duplamente desejável, para ambientes desfavoráveis e favoráveis.

Na Figura 1 encontram-se os padrões de resposta dos seis grupamentos genéticos. Pela inspeção dos diferentes padrões de repostas obtidos, pode-se verificar a importância da interação genótipo x ambientes. Os efeitos ambientais apresentados na abcissa do gráfico foram estimados e demonstram ampla variação de valores. Neste caso, observaram-se ambientes com $900 \mathrm{~kg}$ de produção de leite inferior à média de todos os ambientes e ambientes com 1230 $\mathrm{kg}$ de produção de leite acima deste valor médio. Verificou-se (Figura 1) que o padrão de resposta do grupamento GC2 foi convexo e duplamente desejável e com produção até 305 dias para o melhor ambiente, superior a todos os outros grupamentos genéticos.

$\mathrm{Na}$ Tabela 9, estão apresentadas as estimativas dos índices ambientais para o modelo linear simples e do modelo linear bissegmentado, juntamente com os erros-padrão. Os ambientes desfavoráveis foram aqueles que tiveram estimativas negativas para $o$ índice ambiental, sendo todos aqueles de nível de manejo baixo considerados no presente trabalho para a formação dos 14 ambientes estudados.

Tabela 9 - Estimativa do índice ambiental $(\mathrm{m})$ e respectivo erro-padrão para os 14 ambientes, para o modelo linear simples e bissegmentado de TOLER (1990)

Table 9 - Estimate of environmental index $(m)$ and tandard error to the 14 environments in the linear and bi segmented models of Toler (1990)

\begin{tabular}{cccccc}
\hline $\begin{array}{c}\text { Grupamento genético } \\
\text { Genetic group }\end{array}$ & \multicolumn{2}{c}{$\begin{array}{c}\text { Modelo simples } \\
\text { Linear model }\end{array}$} & & \multicolumn{2}{c}{$\begin{array}{c}\text { Modelo bissegmentado } \\
\text { Bi segmented model }\end{array}$} \\
\cline { 2 - 3 } \cline { 5 - 6 } & $\begin{array}{c}\text { Estimativa } \\
\text { Estimate }\end{array}$ & $\begin{array}{c}\text { Erro-padrão } \\
\text { Standard error }\end{array}$ & & $\begin{array}{c}\text { Estimativa } \\
\text { Estimate }\end{array}$ & $\begin{array}{c}\text { Erro-padrão } \\
\text { Standard error }\end{array}$ \\
\hline 1 & $-780,43^{* *}$ & 102,42 & & $-720,45^{* *}$ & 84,62 \\
2 & $670,41^{* *}$ & 102,38 & & $638,18^{* *}$ & 95,39 \\
3 & $-453,40^{* *}$ & 102,31 & & $-530,45^{* *}$ & 83,69 \\
4 & $420,86^{* *}$ & 102,30 & & $451,94 * *$ & 95,98 \\
5 & $-362,57^{* *}$ & 102,29 & & $-458,16^{* *}$ & 83,86 \\
6 & $1228,07^{* *}$ & 102,67 & & $1215,11^{* *}$ & 97,29 \\
7 & $-533,65^{* *}$ & 102,33 & & $-526,11^{* *}$ & 83,69 \\
8 & $1005,28^{* *}$ & 102,53 & & $985,42^{* *}$ & 95,86 \\
9 & $-564,59^{* *}$ & 102,34 & & $-571,21 * *$ & 83,72 \\
10 & $827,07^{* *}$ & 102,44 & & $878,23^{* *}$ & 95,50 \\
11 & $-819,09^{* *}$ & 102,44 & & $-903,61 * *$ & 87,34 \\
12 & 6,69 & 102,25 & & 39,27 & 92,47 \\
13 & $-898,26^{* *}$ & 102,48 & & $-734,59 * *$ & 84,77 \\
14 & $253,61^{*}$ & 102,27 & & $236,44^{*}$ & 97,39 \\
\hline
\end{tabular}

* significativo a $5 \%$ (Significant $(P<0.05))$

** significativo a $1 \%$ (Significant $(P<0.01))$ 


\section{Conclusões}

O genótipo GC2, por apresentar alta média de produção de leite até 305 dias e ser duplamente desejável de acordo com o padrão de resposta com a variação ambiental, pode ser recomendado para todos os tipos de ambientes.

Somente os grupamentos genéticos GC2 e GC3 poderiam ser recomendados para ambientes desfavoráveis.

Os grupamentos genéticos GC3 e PO possuem resposta média aos ambientes favoráveis, sendo que o PO, por apresentar a maior média de produtividade, pode ser recomendado para ambientes de alto nível de manejo.

$\mathrm{O}$ grupamento genético $\geq \mathrm{GC} 4$ foi considerado duplamente indesejável, sendo desaconselhável a sua recomendação para ambientes favoráveis ou desfavoráveis, apesar da sua alta média de produção.

O grupamento genético $31 / 32$, por fornecer resposta desejável só para ambientes desfavoráveis e apresentar uma das menores produção de leite até 305 dias, não deve ser recomendado. O GC1, apesar de apresentar padrão de resposta média com a variação ambiental, também apresentou baixa média de produção, não devendo ser recomendado para qualquer tipo de ambiente.

\section{Agradecimentos}

Ao CNPq, pelo suporte financeiro, por intermédio da concessão de bolsas de Iniciação Científica e de Pesquisador.

Ao serviço de controle leiteiro da Associação de Criadores de Gado Holandês de Minas Gerais (ACGHMG), pela cessão dos dados que permitiram a realização do presente trabalho.

\section{Referências Bibliográficas}

ARIAS, E.R.A., RAMALHO, M.A.P., FERREIRA, D.F. 1996. Adaptabilidade e estabilidade de cultivares de milho avaliados no Estado de Mato Grosso do Sul. Ciência e Agrotecnologia, 20(4):415-420.

BECKER, H.C., LEON, J. 1988. Stability analysis in plant breeding. Plant Breeding, 101(1):1-23.

CROSSA, J. 1990. Statistical analysis of multilocations trials. Advances in Agronomy, 44:55-85.

CRUZ, C.D., TORRES, R.A. de, VENCOVSKY, R. 1989. An alternative approach to the stability analysis proposed by Silva and Barreto. Rev. Bras. Genet., 12(2):567-80.

EBERHART, S.A., RUSSELL, W.A. 1966. Stability parameters for comparing varieties. Crop Sci., 6(1):36-40.

FERREIRA, D.F., RAMALHO, M.A.P., ABREU, A.F. de B. 1992. Utilização da testemunha na avaliação da estabilidade em ensaios de competição de cultivares. Ciência e Agrotecnologia, 16(3):394-399.

FERREIRA, D.F., ZAMBALDE, A.L. Simplificação de algumas técnicas especiais da experimentação agropecuária no MAPGEN e Softwares correlatos. In: CONGRESSO DA SOCIEDADE BRASILEIRA DE INFORMÁTICA APLICADA A AGROPECUÁRIA E A AGROINDÚSTRIA, SOFTEX 2000, Belo Horizonte, 1997. Anais...Belo Horizonte, p.285-291, 1997.

GALLANT, A.R. 1987. Nonlinear statistical models. New York: John Wiley \& Sons. 610p.

GAUCH JR., H.G., ZOBEL, R.W. 1988. Predictive and postdictive success of statistical analysis of yield trials. Theor. Appl. Genet., 76(1):1-10.

HAMILTON, D., MADEN, J.J.L., COOK, J.P. et al. 1996. Stocking rate, calving season and post-weaning growth effects for Angus cattle on annual pasture. Austr. J. Exp. Agric., 36:401-412.

HOHENBOKEN, W.D. Genetic $\mathrm{x}$ environment interactions and animal production: when nurture and nature collide. REUNIÃO ANUAL DA SOCIEDADE BRASILEIRA DE ZOOTECNIA, SIMPÓSIO INTERNACIONAL SOBRE TÓPICOS ESPECIAIS EM ZOOTECNIA, 33, Fortaleza, 1996. Anais...Fortaleza: SBZ, p.21-34, 1996.

LIN, C.S., BINNS, M.R. A superiority measure of cultivar performance for cultivar x location data. Can. J. Plant Sci., 68:193-198, 1988.

LIN, C.S., BINNS, M.R., LEFKOVICTH, L.P. 1986. Stability analysis: where do we stand? Crop Sci., 26(5):894-900.

PLAISTED, R.L., PATERSON, L.C. 1959.A thecnique for evaluating the ability of selections to yield consistently in different locations or seasons. Amer. Potato J., 36(11):381-385.

RORATO, P.R.N., LÔBO, R.B., FILHO, R.M. et al. 1994. Efeito da interação genótipo $\mathrm{x}$ ambiente sobre a produção de leite da raça Holandesa, no Estado do Paraná. R. Soc. Bras. Zootec., 23(5):859-869.

SAS Institute. SAS language and procedures: Usage. Version 6, 1. ed. Cary NC: SAS Institute, 1995, 373p.

TOLER, J.E. Patterns of genotypic performance over environmental array. Clemson University, USA, 154p. 1990. Thesis (PhD) - Clemson University, 1990.

TOLER, J.E., BURROWS, P.M. 1998. Genotype performance over environmental arrays: A non-linear grouping protocol. J. Appl. Stat., 25(1):131-143.

VERMA, M.M., CHAHAL, G.S., MURTY, B.R. 1978. Limitations of conventional regression analysis: a proposed modification. Theor. Appl. Genet., 53:89-91.

WESTCOTT, B. 1986. Some methods of analyzing genotypeenvironment interactions. Heredity, 56:243-53.
Recebido em: 27/08/98

Aceito em: 02/09/99 